\title{
Logística de transporte: um panorama nacional das empresas de transporte de carga no Brasil
}

\author{
Transportation logistics: a national overview of cargo transportation companies in Brazil \\ Logística de transporte: panorama nacional de las empresas de transporte de carga en Brasil
}

Recebido: 17/06/2021 | Revisado: 24/06/2021 | Aceito: 26/06/2021 | Publicado: 10/07/2021

\author{
João Victor Santana \\ ORCID: https://orcid.org/0000-0002-8627-4023 \\ Universidade do Estado do Pará, Brasil \\ E-mail: victortorsantana@gmail.com \\ Alessandra Xisto Pinheiro \\ ORCID: https://orcid.org/0000-0001-6142-9917 \\ Universidade do Estado do Pará, Brasil \\ E-mail: alessaxisto@gmail.com \\ Breno do Carmo Alexandrino \\ ORCID: https://orcid.org/0000-0003-3106-6478 \\ Universidade do Estado do Pará, Brasil \\ E-mail: brenoalexandrino99@gmail.com \\ Samuel Matheus Casseb da Costa Silva \\ ORCID: https://orcid.org/0000-0001-8552-4054 \\ Universidade do Estado do Pará, Brasil \\ E-mail: samuelmcasseb@gmail.com \\ Heloisa Tembra Bezerra \\ ORCID: https://orcid.org/0000-0001-9785-8465 \\ Universidade do Estado do Pará, Brasil \\ E-mail: heloisa.tb@hotmail.com
}

\begin{abstract}
Resumo
Este estudo tem como objetivo analisar o número de empresas de transporte de cargas ativas no Brasil, sendo comparado o crescimento do número de empresas nas diferentes regiões do país. A pesquisa tem caráter descritivo e exploratório, sendo os dados sobre o quantitativo de empresas de transporte de cargas ativas no Brasil obtidos no Painel Mapa de Empresas, de responsabilidade do Governo Federal. Constatou-se que o país apresentou significativo crescimento na quantidade de empresas ativas entre os anos de 2010 a 2021, sendo a região Sul a que apresentou o maior crescimento, seguida pelas região Sul, Nordeste, Centro-Oeste e Norte, respectivamente. As empresas de transporte de carga por modal rodoviário representam a maior quantidade de empresas de logística de transporte ativas, reforçando que a matriz de transporte brasileira é baseada neste modal. Destaca-se a inexpressiva quantidade de empresas que atuam nos demais modais em comparação com o rodoviário, evidenciando-se a necessidade de ampliação desses modais, com intuito facilitar a integração entre todos.
\end{abstract}

Palavras-chave: Logística; Desenvolvimento regional; Modais de transporte; Operadores logísticos.

\begin{abstract}
This study aims to analyze the number of active cargo transport companies in Brazil, comparing the growth in the number of companies in different regions of the country. The research is descriptive and exploratory, with data on the number of active cargo transport companies in Brazil obtained from the Map of Companies Panel, under the responsibility of the Federal Government. It was found that the country showed a significant growth in the number of active companies between the years 2010 to 2021, with the South region showing the greatest growth, followed by the South, Northeast, Midwest and North regions, respectively. Cargo transport companies by road modal represent the largest number of active transport logistics companies, reinforcing that the Brazilian transport matrix is based on this modal. The inexpressive number of companies operating in other modes stands out compared to road transport, highlighting the need to expand these modes, in order to facilitate integration among all.
\end{abstract}

Keywords: Logistics; Regional development; Modes of transport; Logistics operators.

\section{Resumen}

Este estudio tiene como objetivo analizar el número de empresas de transporte de carga activas en Brasil, comparando el crecimiento en el número de empresas en diferentes regiones del país. La investigación es descriptiva y exploratoria, con datos sobre el número de empresas de transporte de carga activas en Brasil obtenidos del Panel 
Mapa de Empresas, bajo la responsabilidad del Gobierno Federal. Se encontró que el país mostró un crecimiento significativo en el número de empresas activas entre los años 2010 a 2021, siendo la región Sur la que mostró el mayor crecimiento, seguida de las regiones Sur, Noreste, Medio Oeste y Norte, respectivamente. Las empresas de transporte de carga por modalidad vial representan el mayor número de empresas logísticas de transporte activas, reforzando que la matriz de transporte brasileña se basa en este modal. Destaca el inexpresivo número de empresas que operan en otros modos en comparación con el transporte por carretera, lo que resalta la necesidad de ampliar estos modos, con el fin de facilitar la integración entre todos.

Palabras clave: Logística; Desarrollo regional; Modos de transporte; Operadores logísticos.

\section{Introdução}

O avanço da globalização, da tecnologia de produção e da comunicação têm tornado a cultura de consumo cada dia mais enraizada na sociedade, refletindo em como o ato de consumir ultrapassou a característica de mero fenômeno de produção e distribuição de bens materiais e passou a difundir símbolos e signos carregados pelas mercadorias (Cichelero \& Galiotto, 2020). Neste cenário de mudanças, Khotamov e Ismoilov (2020) dizem que os países exportam e importam para obter economias de escala na produção, já que se produzirem apenas uma gama limitada de bens, poderão produzir cada um desses bens em maior escala e com mais eficiência do que se tentasse produzir tudo.

O Brasil apresenta significativas quantidades de produtos exportados e de importados, que são representados, respectivamente, por produtos primários e por manufaturados, sendo estas atividades imprescindíveis para o contínuo desenvolvimento econômico do país (Gelatti et al., 2020). Neste cenário, tem-se a logística de transporte como fator imprescindível, pois possibilita que matérias-primas e bens de consumo atravessem distâncias continentais e cheguem por meio de diferentes modais ao seu destino (Viveros et al., 2020).

De Souza e Bouchut (2017) constataram que o índice de desempenho logístico brasileiro apresentou uma leve evolução nos últimos anos, se aproximando da média encontrada para o grupo de médio desempenho logístico, formado por países que são os seus concorrentes diretos na atividade exportadora. Entretanto, segundo Nakamura (2018), a infraestrutura logística do país não tem se desenvolvido em acordo com o crescimento com setores que exportam e importam produtos primários, fazendo com que o país viva em estado de caos logístico e de infraestrutura.

Da Costa e Marjotta-Maistro (2017) dizem que no sentido amplo da discussão sobre logística, o Brasil precisa de investimentos em outros modais logísticos, tendo em vista aumentar o volume de cargas transportada e barateando o custo do transporte, já que a concentração do modal rodoviário torna-se um problema, considerando-se que os indicadores de qualidade que apontam para uma maioria de rodovias com problemas. Para além da visão a nível global e nacional, é preciso observar a estrutura da logística regional, que é responsável por deslocar esses produtos do local de produção até o centro de distribuição e que indica mudanças nas escalas de produção e nas relações sociais (Santos \& Proença, 2020).

Tendo em vista a gestão de custos logísticos (logistics cost management - LCM), Franco \& Gasparetto (2020) realizaram um estudo com grandes empresas industriais localizadas na Colômbia e concluíram que parte das empresas estudadas possui logística terceirizada, seguindo tendência mundial de transferir essas atividades para outras instituições, e também há as não possuem área de logística formalmente constituída. Tratando especificamente do transporte de última milha, Souza et al. (2020) dizem que países desenvolvidos economicamente tendem a buscar soluções mais sofisticadas através uso de veículos tecnologicamente inovadores, enquanto os menos desenvolvidos apostam em soluções com menor nível de tecnologia associada, de fácil adoção e baixa necessidade de investimentos financeiros.

Ademais, ao avaliar os principais gargalos logísticos, Nunes, de Oliveira e Vacca (2020) identificaram situações que envolvem estruturas precárias de transporte, ausência de políticas público-privadas e ineficiência de processos, aumentando ainda mais o tempo de execução do transporte. Sendo assim, torna-se necessário o aprimoramento dos processos de transportes 
já existentes, bem como a identificação de novas oportunidades de mercado advindas da área de logística (Lima, Schuchmann \& Figueira, 2020; Crevatin \& Zilber, 2020).

Nessa perspectiva, observa-se um cenário favorável à implantação e aprimoramento de empresas que atuam prestando serviços de transporte de carga, uma vez que, conforme mencionado anteriormente, existe a predisposição das empresas industriais transferirem esse serviço para operadores logísticos. Face ao exposto, este estudo tem como objetivo analisar o número de empresas de transporte de cargas ativas no Brasil, sendo comparado o crescimento do número de empresas nas diferentes regiões do país.

\section{Metodologia}

Esta pesquisa tem caráter descritivo, que registra e descreve os fatos observados sem interferir neles, e também é exploratória, sendo realizada pesquisa bibliográfica e estudos de caso, com levantamento de informações que poderão, conforme Oliveira (2011), levar o pesquisador a conhecer mais a respeito do tema proposto. Essa metodologia foi complementada com o levantamento de dados documentais em sites de busca aberta.

No que se refere aos dados sobre o quantitativo de empresas de transporte de cargas ativas no Brasil, estes foram obtidos no Painel Mapa de Empresas, de responsabilidade do Governo Federal. Para esta pesquisa, foram consideradas apenas as empresas que trabalham com transporte de cargas que estão ativas, incluindo perigosas, por meio de diferentes modais, não sendo consideradas as que trabalham com transporte de passageiros ou mudanças.

Primeiramente, considerou-se o quantitativo total de empresas de transporte de carga ativas no Brasil entre o período de 2010 a maio de 2021 e então pesquisou-se pelas regiões brasileiras, considerando-se apenas o quantitativo de empresas ativas em maio de 2021, sendo selecionados blocos com os estados que formam cada uma das regiões brasileiras. Por seguinte, coletou-se os dados dividindo as empresas entre diferentes modais de transporte. Então, os dados coletados foram tratados através de um software de planilhas eletrônicas, para facilitar a visualização e compreensão dos mesmos.

\section{Resultados e Discussão}

Os dados apresentados na Figura 1 são referentes à quantidade de empresas de transporte de cargas ativas no Brasil e nas cinco regiões brasileiras. Estes dados se referem ao quantitativo total de empresas de transporte de carga que trabalham com diferentes modais de transporte, sendo considerados modais rodoviários, ferroviários, aquaviários, aéreos e dutoviários. Assim, é possível observar que o país saiu de 106.754 empresas de transporte em 2010 e chegou ao expressivo número de 474.340 em maio de 2021, o que representa um crescimento de $344,32 \%$ neste intervalo de tempo.

Em uma perspectiva por região, evidencia-se que a região sudeste é a que apresenta o maior percentual de empresas ativas em comparação com as demais regiões e que a norte é a que apresenta a menor quantidade a nível nacional. Ressalta-se que em 2010 a região sul possuía um quantitativo próximo ao da região sudeste, mas ao longo dos anos elas foram se distanciando, sendo observado que na região sudeste houve um crescimento no número de empresas maior do que na região sul. As demais regiões também apresentaram um crescimento contínuo, mas menos acelerado do que as regiões sul e sudeste. 
Figura 1 - Quantidade de empresas de transporte de cargas, incluindo perigosas, que atuam com diferentes modais ativas no Brasil e em cada região brasileira.

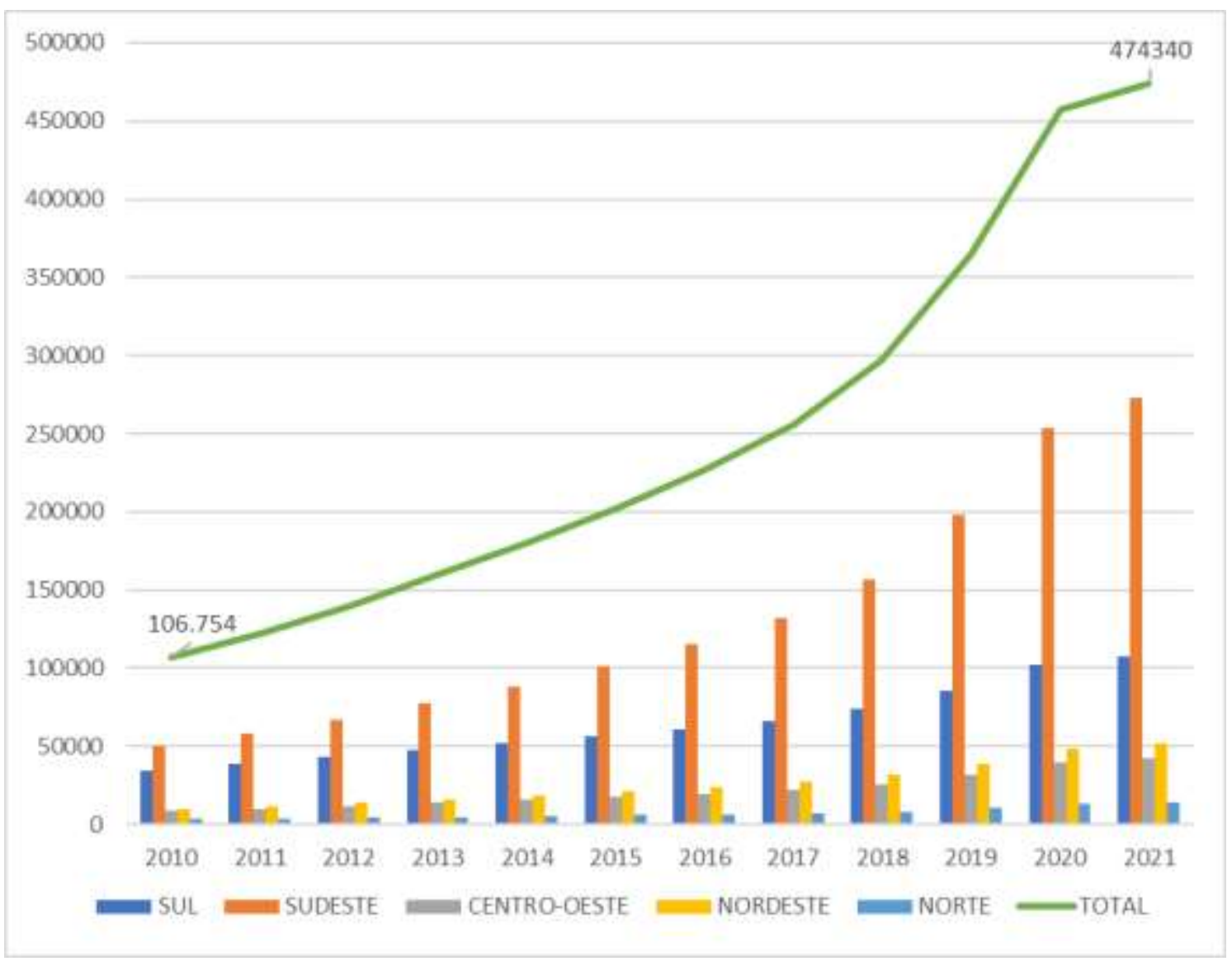

Fonte: Painel Mapa de Empresas, elaborado pelo autor (2021).

Pereira, Santos e Ferreira (2019), ao tratarem sobre o comércio de commodities, comentam que os três principais portos brasileiros estão localizados nas regiões Sul e Sudeste, sendo pioneiras na instalação de empresas processadoras e na implantação de sistemas de armazenagem, que expandiram os serviços logísticos e atraíram grande parte dos fluxos de produtos agrícolas e seus derivados da região Centro-Oeste do Brasil. Tendo em vista o supracitado, infere-se que ao fato das regiões Sul e Sudeste apresentarem a maior porcentagem de empresas de transporte de carga não está relacionada somente com a demanda de fluxo de carga nessas regiões, mas também com a precursão na implantação de empresas desse ramo.

Ademais, ao analisar a dinâmica geoeconômica da comercialização, logística e transporte da cadeia produtiva do leite na região sul, Camilor (2018) conclui que as tecnologias aplicadas aos sistemas de transporte representam um significativo avanço nos aspectos conjunturais e estruturais deste segmento, sendo observado o uso caminhões híbridos para o transporte de diferentes mercadorias em diferentes temperaturas, redução de gastos fixos e potencialização da capacidade de carga. Em relação à logística de modo geral, o autor evidenciou que os avanços não estão somente na organização da rota a ser percorrida, mas também no tratamento das mercadorias em grandes Centros de Distribuição - CD.

Ainda de acordo com o gráfico 1, as regiões Nordeste e Centro-Oeste ocupam, respectivamente, a terceira e a quarta posição no quantitativo de empresas ativas em comparação com as cinco regiões, sendo possível observar que estas duas regiões apresentaram um crescimento semelhante durante os anos tratados nesta pesquisa. Também se observa que a região norte é a que apresenta a menor quantidade de empresas ativas, tendo apresentado um crescimento contínuo, mas menos acelerado do que as demais regiões. 
Conforme os dados apresentados pelo Diário Econômico ETENE (Freire, 2020), todos os estados da região Nordeste do Brasil têm apresentado efetiva atividade de exportação e importação, destacando-se o estado da Bahia, que possui os maiores quantitativos da atividade. Nesse sentido, é válido trazer o estudo de Silva, Bertoncini e Silva (2017), que realizaram uma análise do papel de investimentos em infraestrutura de transporte sobre o desenvolvimento econômico de uma amostra de municípios localizados em Pernambuco, e concluíram que a promoção de uma obra de infraestrutura de transporte ferroviário teve potencial para induzir o aumento de bem-estar da sociedade em geral e desenvolvimento econômico na região.

Segundo Silva (2020), a agricultura e a indústria de produtos minerais não metálicos são as atividades mais representativas para o desenvolvimento regional do Centro-Sul brasileiro, tendo a agricultura apresentado um forte e espalhado crescimento por toda a região. Nesse cenário, a logística de transporte é relevante para o escoamento da produção do agronegócio da região, sendo utilizado principalmente o modal rodoviário para o transporte da produção até os recintos alfandegados, localizados nas regiões Sudeste, Sul, Nordeste e Norte do Brasil, uma vez que os estados dessa região escoam boa parte de sua produção pelos portos (Pais \& Torres, 2018; Santos \& Pereira, 2019).

A região Norte apresenta crescente atividade de importação e exportação, principalmente por causa dos estados do Amazonas, responsável pelas importações de produtos manufaturados, e do Pará, que é o maior responsável pela exportação de minérios (Lobão, Corrêa \& Schneider, 2017). Contudo, conforme pode ser observado no gráfico 1, esta região é a que apresenta a menor quantidade de empresas de transporte de cargas no Brasil, sendo válido ressaltar que a região também foi a que apresentou o menor crescimento ao longo dos anos, o que a distanciou das demais regiões.

Corroborando com as afirmações dos autores supracitados, os resultados apresentados na tabela 1 demonstram que mais da metade das empresas de transporte de cargas ativas no Brasil em maio de 2021 são do modal rodoviário. Nacionalmente, o modal hidroviário é o que apresenta o segundo maior quantitativo de empresas abertas, seguido por aéreo, dutoviário e ferroviário. É válido ressaltar a diferença expressiva entre a quantidade de empresas do modal rodoviário (471.511) para o hidroviário (2.217), aéreo (252), dutoviário (189) e ferroviário (171), que evidencia a necessidade de investimentos nesses modais.

Tabela 1 - Quantidade de empresas de transporte de carga por diferentes modais e regiões brasileiras ativas em maio de 2021.

\begin{tabular}{cccccc}
\hline & Dutoviário & Aéreo & Rodoviário & Hidroviário & Ferroviário \\
\hline Norte & $10(5,29 \%)$ & $33(13,09 \%)$ & $13.619(2,89 \%)$ & $898(40,50 \%)$ & $12(7,01 \%)$ \\
Nordeste & $43(22,75 \%)$ & $56(22,22 \%)$ & $44.198(9,38 \%)$ & $208(9,39 \%)$ & $21(12,28 \%)$ \\
Centro-Oeste & $15(7,94 \%)$ & $22(8,73 \%)$ & $43.043(9,13 \%)$ & $105(4,74 \%)$ & $21(12,28 \%)$ \\
Sudeste & $99(52,38 \%)$ & $110(43,65 \%)$ & $261.959(55,55 \%)$ & $763(34,41 \%)$ & $65(38,01 \%)$ \\
Sul & $22(11,64 \%)$ & $31(12,30 \%)$ & $108.701(23,05 \%)$ & $243(10,96 \%)$ & $52(30,04 \%)$ \\
\hline Total & $189(100 \%)$ & $252(100 \%)$ & $471.511(100 \%)$ & $2.217(100 \%)$ & $171(100 \%)$ \\
\hline
\end{tabular}

Fonte: Painel Mapa de Empresas, elaborado pelos autores (2021).

Analisando sob a perspectiva regional, observa-se que a região sudeste apresenta as maiores porcentagens em quatro dos cinco modais, o que já era previsto tendo em vista os dados apresentados no Gráfico 1. Assim, observando os quantitativos apresentados na Tabela 1, considera-se que o quantitativo de empresas do modal rodoviário é o que justifica os resultados apresentados no Gráfico 1.

Considerando a quantidade atual de empresas ativas em maio de 2021 (474.340), é válido considerar o Plano Nacional de Logística e Transporte (MT, 2018), que diz que a matriz de transporte do Brasil é composta majoritariamente por modais rodoviários, o que traz grandes desvantagens econômicas para o país quando se fala em competitividade internacional, já que o gasto com transporte rodoviário representa cerca de $20 \%$ do PIB. Neste cenário, as condições de infraestrutura das rodovias brasileiras configuram-se como importante fator para o bom funcionamento, segurança e melhoria das condições de trânsito, já 
que com a crescente demanda do tráfego, principalmente por veículos pesados, é necessário encontrar uma pavimentação de alta durabilidade, baixo custo de manutenção e maior segurança para os usuários (Carmo \& Raia, 2018; Silva et al. 2020).

Barreto e Ribeiro (2020) dizem que, diferentemente do Brasil, em outros países de dimensões continentais, é possível notar que há uma preferência pela utilização do modal ferroviário, se aproveitando deste para transportar grandes volumes a longas distâncias. Aurélio Neto (2018) também ressalta a necessidade de reorientar a matriz de transporte para uma maior intermodalidade entre o modal rodoviário e ferroviário, sendo o modal ferroviário mais adequado para o transporte de carga por longas distâncias, desde os pátios da ferrovia até os portos litorâneos. Outrossim, Dantas e Fraga (2021), concluem que se o Brasil colocar em prática os projetos ferroviários, ampliando os investimentos no modal e buscando contratos viáveis de concessão, poderá explorar de maneira eficaz sua enorme potencialidade em desenvolver uma importante e coesa malha ferroviária.

É válido ressaltar que, conforme Aquino et al. (2018), as rodovias, não necessariamente, receberão todo o acréscimo na quantidade de tráfego, já que há a tendência de criação de novas rotas comerciais com a construção de novas estradas. Conduto, Nunes (2018) comenta que o Brasil levará meio século para atingir o nível de infraestrutura de países desenvolvidos, uma vez que as obras de criação e ampliação de rodovias, ferrovias, portos e aeroportos iniciadas com o Programa de Aceleração do Crescimento não foram concluídas.

A região Norte é a que possui a maior quantidade de empresas do modal hidroviário (tabela 1), mas esta quantidade ainda se encontra muito distante do potencial que a região tem para este modal, já que é diagnosticada baixa eficiência neste setor na região, podendo essa ineficiência ser atribuída às questões ambientais (Barros \& Almeida, 2019). O transporte hidroviário na região não tem avançado em um século, já que os investimentos na área são ínfimos, e as perdas de mercadorias continuam altas para os comerciantes, que acabam elevando os preços dos produtos para cobrir as suas perdas (Grisotti \& Moran, 2020). Infere-se que este fato esteja ligado às questões geográficas e culturais da região onde são observados conflitos entre se desenvolver e preservar a floresta Amazônica, juntamente com as terras indígenas, comunitárias e unidades de conservação (Andrade, 2018).

No que tange ao modal dutoviário, Bovolenta e Biaggioni (2019) dizem que as rotas dutoviárias são mais sustentáveis energeticamente, não sendo diretamente dependente de combustíveis fósseis, como os demais modais. Entretanto, diversas são as barreiras para a ampliação da área, como os direitos de acesso podem limitar o exercício legal do direito de uso pelo operador de dutos, falta de uma definição dos princípios e da metodologia de reajuste tarifário dos serviços de transporte dutoviário e a complexidade no plano de manutenção, visando à operação segura das instalações (Braga \& Leister, 2018).

Considerando ainda a Tabela 1, percebe-se que o modal aéreo também é pouco explorado a nível nacional, estando mais concentrado na região Sul. Segundo Castro (2018), a ampliação dos modais aéreos não deve gerar muitos impactos na economia local, mas apresenta um potencial relevante a ser explorado referente ao comércio externo. Almeida et al. (2019) ressaltam a necessidade de o governo brasileiro olhar com mais atenção para a situação dos modais aéreos, uma vez que todo o sistema sente as consequências negativas da ausência da aviação, que poderia transportar mais cargas, consequentemente, contribuir com a economia.

Ademais, Schyra (2019) constata a importância das modalidades de transporte para as empresas e também para a gestão governamental, bem como ressalta a necessidades de investimentos voltados para diversificação dos modais logísticos, já que são necessários modais que se adequem às especificidades das cargas transportadas. Ferigato e da Silva (2021) também afirmam que durante a escolha de qual modal é mais adequado para se transportar determino tipo de carga, deve-se considerar as principais características da mesma e também as necessidades do embarcador. Outrossim, Gama, Silva e Costa (2019) mostram que não existe um modal de transporte melhor do que outro, já que a via de transporte se torna mais apropriada de acordo com sua finalidade, sendo necessário se pensar na integração dos modais a nível nacional. 


\section{Considerações Finais}

O presente estudo teve como objetivo analisar o número de empresas de transporte de cargas ativas no Brasil, sendo analisado diferentes regiões do país e por diferentes modais de transporte. Assim, foi possível constatar que o país apresentou significativo crescimento na quantidade de empresas ativas entre os anos de 2010 a 2021, sendo a região Sul a que apresentou o maior crescimento, seguida pelas região Sul, Nordeste, Centro-Oeste e Norte, respectivamente. Considerando os estudos de outros autores, conclui-se que a quantidade de empresas de cada região está ligada a forma como se deu o desenvolvimento regional dos estados, bem como aos investimentos em infraestrutura das regiões.

Constatou-se que as empresas de transporte de carga por modal rodoviário representam a maior quantidade de empresas de logística de transporte ativas, reforçando que a matriz de transporte brasileira é baseada neste modal. Destaca-se a inexpressiva quantidade de empresas que atuam nos demais modais em comparação com o rodoviário, evidenciando-se a necessidade de ampliação desses modais, com intuito facilitar a integração entre todos.

Por fim, considerando a importância da atividade de exportação e importação para a economia brasileira, ressalta-se a necessidade de contínuas pesquisas relacionadas ao setor de logística, visando identificar as expectativas e realidades do setor. Outrossim, também se faz necessário analisar o quantitativo das empresas a nível estadual e pelos modais individualmente, tendo em vista as especificidades de cada estado e modal. Assim, sugere-se que sejam realizadas pesquisas sobre estruturas logísticas no estado de São de Paulo, tendo em vista que este é o estado mais desenvolvido logisticamente no Brasil.

\section{Referências}

Almeida, J. V. P. et al. (2019). Infraestrutura aeroportuária brasileira: uma análise do modal. Revista Científica, 1 (1).

Andrade, R. P. (2018). A poeira do progresso pede passagem: imagens de natureza e desenvolvimento na floresta amazônica. Anais do Museu Paulista: História e Cultura Material, 26. https://doi.org/10.1590/1982-02672018v26e14

Aquino, T. S. A. et al. (2018). Avaliação funcional e operacional de rodovias federais brasileiras. Anais do $32^{\circ}$ Congresso de Pesquisa e Ensino e Transporte. Gramado, SC, Brasil.

Aurélio Neto, O. (2018). O Brasil no mercado mundial de carne bovina: análise da competitividade da produção e da logística de exportação brasileira. Ateliê Geográfico, 12(2), 183-204. https://doi.org/10.5216/ag.v12i2.47471

Barreto, R. C. P., \& Ribeiro, A. J. M. (2020). Logística no Brasil: uma análise do panorama dos modais rodoviários e ferroviários no cenário nacional demonstrando as vantagens e desvantagens das referidas modalidades. Revista Livre de Sustentabilidade e Empreendedorismo, 5(3), $145-176$.

Barros, G. O., \& de Almeida, E. S. (2019). Mensuração da eficiência portuária na região norte aplicando a análise envoltória dos dados (DEA). Exacta, 17 (2). https://doi.org/10.5585/exactaep.v17n2.8004

Bovolenta, F. C., \& Biaggioni, M. A. M. (2019). Resultados energéticos da Logística e Transporte do Etanol. Revista AgroFIB, 1 (1).

Braga, A. C., \& Leister, A. C. C. C. (2018). Uma análise do mercado do transporte dutoviário de petróleo seus derivados e gás natural no Brasil. Caminhos de Geografia, 19 (67), 234-252. https://doi.org/10.14393/Hygeia196716

Camilor, P. J. (2018). A dinâmica geoeconômica da comercialização, logística e transporte da cadeia produtiva do leite na Região Sul do Brasil. Tese de doutorado em Geografia - Centro de Filosofia e Ciências Humana, Universidade Federal de Santa Catarina, SC, Brasil.

Carmo, C. L., \& Raia, A. A. R. (2018). Avaliação das condições de infraestrutura viária em trechos urbanos críticos das rodovias federais brasileiras. Anais do $32^{\circ}$ Congresso de Pesquisa e Ensino e Transporte.

Castro, L. M. O. (2018). Conectividade aérea e comércio exterior: os efeitos do hub aéreo nas exportações e as repercussões na economia cearense. Dissertação de Mestrado em Economia do Setor Público - Faculdade de Economia, Administração, Atuária e Contabilidade, Universidade Federal do Ceará UFC.

Cichelero, C. A., \& Galiotto, R. (2020). Sociedade de consumo e crise ambiental: os reflexos do hiperconsumo. Biodiversidade, recursos hídricos e direito ambiental, 112. Educs.

Costa, M. S., \& Marjotta-Maistro, M. C. (2017). Indicadores de qualidade da infraestrutura logística brasileira: um estudo para o agronegócio. Revista Brasileira de Iniciação Científica, 4 (9).

Crevatin, G., \& Zilber, S. N. (2020). Projeto de inovação em um processo logístico no contexto da economia compartilhada: Vehicle fill rate. Revista de Gestão e Projetos, 11(3), 46-71. https://doi.org/10.5585/gep.v11i3.18580 
Dantas, A. A. N., \& Fraga, Y. S. B. (2021). Ferrovias no Brasil: Projetos futuros e em andamento. Research, Society and Development, 10(5). https://doi.org/10.33448/rsd-v10i5.14917

Ferigato, E., \& Silva, D. D. C. (2021). Os modais de transporte de carga no Brasil. RECIMA21-Revista Científica Multidisciplinar,2(2). https://doi.org/10.47820/recima21.v2i2.94

Franco, M. A. J., \& Gasparetto, V. (2020). Práticas para a gestão de custos logísticos em empresas industriais de grande porte da Colômbia. Estudios Gerenciales, 364-373. https://doi.org/10.18046/j.estger.2020.156.3754

Freire, L. L. R. (2020). Exportações e importações dos Estados do Nordeste até setembro de 2020. Diário Econômico ETENE, Banco do Nordeste do Brasil, Fortaleza, CE, Brasil.

Gama, K. N. C., Silva, L. R., \& Costa, T. S. (2019). Integração dos modais de transportes frente ao desenvolvimento econômico brasileiro. Research, Society and Development, 8 (9). http://dx.doi.org/10.33448/rsd-v8i9.1330

Gelatti, E. et al. (2020). Desindustrialização no Brasil: uma análise à luz das exportações e importações (1997 a 2018). RDE-Revista de Desenvolvimento Econômico, 1(45). http://dx.doi.org/10.36810/rde.v1i45.6379

Grisotti, M., \& Moran, E. F. (2020). Os novos desafios do desenvolvimento na região amazônica. Civitas-Revista de Ciências Sociais, 20(1). https://doi.org/10.15448/1984-7289.2020.1.36617

Khotamov, I., \& Ismoilov, A. (2020). Is estimating and forecasting trends of global export and import of goods in international markets. Aрхив научных исследований. https://tsue.scienceweb.uz/index.php/archive/article/view/1355

Lima, M. A., Schuchmann, B. M., \& Figueira, A. A. (2020). Aprimoramento do transporte de produtos paletizados em caminhões. Brazilian Journal of Business, 2(4), 3975-3985. https://doi.org/10.34140/bjbv2n4-032

Lobão, M. S. P.; Corrêa, A. S. \& Schneider, M. B. Região Norte do Brasil e sua inserção no comércio internacional brasileiro. Interações (Campo Grande), 18 (2), p. 87-101, 2017. https://doi.org/10.20435/inter.v18i2.1433

MT (2018). Plano Nacional de Logística e Transportes 2015. Brasília: Ministério dos Transportes, Brasil. Recuperado de: https://www.ontl.epl.gov.br/html/objects/_downloadblob.php?cod_blob=5835

Nakamura, A. L. D. S. (2018). Infraestrutura de transportes como instrumento estatal de promoção do desenvolvimento econômico e social. Tese de Doutorado em Direito Político e Econômico - Universidade Presbiteriana Mackenzie, São Paulo, Brasil.

Nunes, S. F., de Oliveira, F. H., \& Vacca, A. C. (2020). Gargalos logísticos de importação: estudo de caso em um distribuidor farmacêutico. South American Development Society Journal, 5(15), 34. http://dx.doi.org/10.24325/issn.2446-5763.v5i15p34-50

Nunes, M. H. (2018). Estudo da logística de transporte de cargas no Sul do Brasil. Negócios em projeção, 9(2), 91-99.

Oliveira, M. F. (2011). Metodologia científica: um manual para a realização de pesquisas em administração. Manual para pós-graduação.

Pais, J. M. \& Torres, C. E. G. (2018). Logística de transportes e expansão da produção de soja no Centro oeste. Revista de Economia do Centro-Oeste, Goiânia, 4 (2).

Pereira, L. A. G., dos Santos, I. J. F., \& Ferreira, W. R. (2019). Geografia do comércio de commodities, dinâmicas espaciais da logística de transportes e dos fluxos de exportações do setor de soja no Brasil. Geografia Ensino \& Pesquisa, 23 (3). https://doi.org/10.5902/2236499433183

Santos, W. R., \& Proença, A. D. (2020). A infraestrutura rodoviária e a urbanização regional contemporânea no território paulista: o caso do corredor urbano Campinas-Sorocaba, Brasil. EURE (Santiago), 46(138), 235-256. http://dx.doi.org/10.4067/S0250-71612020000200235

Santos, J. D. S., \& Pereira, L. A. G. (2019). Logística de transportes do agronegócio e exportações de soja no Centro-Oeste brasileiro. Geoambiente On-Line, (34), 131-154. https://doi.org/10.5216/revgeoamb.v0i34.52867

Schyra, L. (2019). Diversificação dos modais de transporte no Brasil. Revista de estudos em Linguagens e Tecnologia, 18(1).

Silva, P. C. S. M. et al. (2020). Avaliação das condições de superfície de pavimento rígido na Rodovia BR-232 e correlação com o índice de acidentes rodoviários. Brazilian Journal of Development, 6(10). https://doi.org/10.34117/bjdv6n10-441

Silva, I. T. (2020). O modelo base exportação para os municípios do Centro-Oeste brasileiro. Dissertação (Mestrado em Economia) - Universidade Estadual de Ponta Grossa, Ponta Grossa, Brasil.

Silva, E. V. A.; Bertoncini, B. V.; Silva, F. G. F. (2017). Infraestrutura de transporte e desenvolvimento econômico: Uma análise a partir da construção da ferrovia transnordestina. Revista Espacios, 38 (47).

Souza, C. D. O. et al. (2020). Soluções para o transporte urbano de cargas na etapa de última milha. urbe. Revista Brasileira de Gestão Urbana, 12 . https://doi.org/10.1590/2175-3369.012.e20190138

Souza, Â. R. L., \& Bouchut, M. C. L. (2017, November). Custos Logísticos no Brasil: avaliação do desempenho logístico brasileiro no comércio internacional na última década (2007-2016). Anais do $27^{\circ}$ Congresso Brasileiro de Custos - ABC, Florianópolis, SC, Brasil.

Viveros, L. J. H. et al. (2020). Estrategia de la mejora continua de la logística de transporte, una evaluación del diseño de una red de conexión continental multimodal de transporte, caso: Ruta Panamericana. Inclusión \& Desarrollo, 7(1). 